\title{
ARTICLE
}

Received 7 May 2014 | Accepted 14 Nov 2014 | Published 28 Jan $2015 \quad$ DOl: 10.1038/ncomms7005

\section{Topological superconductivity and unconventional pairing in oxide interfaces}

\author{
Mathias S. Scheurer ${ }^{1} \&$ Jörg Schmalian ${ }^{1,2}$
}

Pinpointing the microscopic mechanism for superconductivity has proven to be one of the most outstanding challenges in the physics of correlated quantum matter. Thus far, the most direct evidence for an electronic pairing mechanism is the observation of a new symmetry of the order parameter, as done in the cuprate high-temperature superconductors. Alternatively, global, topological invariants allow for a sharp discrimination between states of matter that cannot be transformed into each other adiabatically. Here we propose an unconventional pairing state for the electron fluid in two-dimensional oxide interfaces and establish a direct link to the emergence of non-trivial topological invariants. Topological signatures, in particular Majorana edge states, can then be used to detect the microscopic origin of superconductivity. In addition, we show that also the density wave states that compete with superconductivity sensitively depend on the nature of the pairing interaction.

\footnotetext{
${ }^{1}$ Institut für Theorie der Kondensierten Materie, Karlsruher Institut für Technologie, D-76131 Karlsruhe, Germany. ${ }^{2}$ Institut für Festköperphysik, Karlsruher Institut für Technologie, D-76131 Karlsruhe, Germany. Correspondence and requests for materials should be addressed to M.S.

(email: mscheurer@tkm.uni-karlsruhe.de).
} 
T he two-dimensional (2D) electron fluid that forms ${ }^{1}$ at the interface between the insulators $\mathrm{SrTiO}_{3}$ and $\mathrm{LaAlO}_{3}$ is an example of an engineered quantum system, where a new state of matter emerges as one combines the appropriate building blocks. The subsequent discovery of superconductivity ${ }^{2}$ in the interface, along with the ability to control the ground state via applied electric fields ${ }^{3}$ opened up intense research. The key open question is whether electronic correlations promote novel phases, such as unconventional superconductivity or exotic magnetically ordered states ${ }^{4-8}$ and how such phases are related to each other.

Generally speaking, new states of matter can be sharply distinguished from conventional behaviour when they break a symmetry or differ in their topology. An example for the former case are the cuprate high-temperature superconductors ${ }^{9,10}$. The second category consists of topological insulators and superconductors, which have recently had a major impact on solid state physics ${ }^{11,12}$. Here the Bloch Hamiltonian, a mapping from momentum space to the space of Hamiltonians, is not adiabatically connected to the vacuum.

In this paper, we establish a direct connection between pairing mechanism and topology of the superconducting state at the interface: Unconventional pairing, that is superconductivity based on the exchange of particle-hole excitations, leads to a topological phase with Majorana bound states and related non-trivial topological aspects. More specifically, we find a time-reversal preserving topological superconductor that has attracted recent attention $^{13-18}$. In contrast, conventional electron-phonon coupling in the same system would yield a topologically trivial state. Consequently, our results allow to identify the unresolved microscopic mechanism of superconductivity in oxide interfaces by probing the topological properties of the pairing state. We also study competing phases, expected to emerge nearby superconductivity in the phase diagram. For a conventional pairing mechanism we find charge density wave order, while an in-plane spin density wave with magnetic vortices competes with unconventional superconductivity.

\section{Results}

Interacting low-energy model. It is well known that the relevant low-energy degrees of freedom at the interface are made up of the titanium $3 d_{x y}, 3 d_{x z}$ and $3 d_{y z}$ orbitals ${ }^{19,20}$. There has been an ongoing debate about why the concentration of transport carriers is considerably lower than what is seen in local charge measurements or expected from the polar catastrophe. One widely accepted explanation ${ }^{21-23}$ is that the electrons in the $3 d_{x y}$ states, that are spatially much closer to the interface than the other orbitals, are mainly localized, whereas the electrons occupying the $3 d_{x z}$ and $3 d_{y z}$ orbitals are delocalized. It indicates that the key electrons at the interface, at least for understanding superconductivity, reside in the $3 d_{x z}$ and $3 d_{y z}$ states. This picture is confirmed by the experimental observation that the emergence of superconductivity is correlated with the chemical potential entering the $3 d_{x z}$ and $3 d_{y z}$ orbitals ${ }^{24,25}$.

Thus, we assume that the crucial states near the Fermi energy of the oxide interface are made up of titanium $3 d_{x z}$ and $3 d_{y z}$ orbitals. The orientation of the electron clouds leads to a wave function overlap along the $x$ direction that is much larger for $3 d_{x z}$ states compared with $3 d_{y z}$, and vice versa for the $y$ direction. Each orbital is then characterized by a light mass $m_{1}$ and a heavy mass $m_{\mathrm{h}}$, leading to the experimentally observed strongly anisotropic electronic structure ${ }^{19,20}$. For example, the energy of the $3 d_{x z}$ states can be described by

$$
\varepsilon_{x z}(\boldsymbol{k})=\frac{k_{x}^{2}}{2 m_{\mathrm{l}}}+\frac{k_{y}^{2}}{2 m_{\mathrm{h}}},
$$

where $m_{\mathrm{h}} / m_{1} \simeq 15 \ldots 30 . \quad \varepsilon_{y z}$ follows from equation (1) by interchanging $k_{x}$ and $k_{y}$. In addition, the electronic properties of the polar interface between insulating oxides is strongly affected by the spin-orbit interaction. Due to the DresselhausRashba effect ${ }^{26,27}$, the electronic states experience a momentumdependent splitting and mixing of spin states, naturally explaining magnetotransport experiments ${ }^{28,29}$. The effect might also be responsible for the observed phase separation in the interfaces ${ }^{30}$. Focusing on the $3 d_{x z}$ and $3 d_{y z}$ states, the most general form up to linear order in momentum that is consistent with the $C_{4 v}$-point-group symmetry and time-reversal invariance is given by

$$
\begin{aligned}
H_{\mathrm{so}}(\boldsymbol{k})= & \frac{1}{2} \lambda \tau_{2} \sigma_{3}+\alpha_{0} \tau_{0}\left(k_{x} \sigma_{2}-k_{y} \sigma_{1}\right) \\
& +\alpha_{1} \tau_{1}\left(k_{x} \sigma_{1}-k_{y} \sigma_{2}\right)+\alpha_{3} \tau_{3}\left(k_{x} \sigma_{2}+k_{y} \sigma_{1}\right),
\end{aligned}
$$

where the Pauli matrices $\sigma_{i}$ and $\tau_{j}(i, j=0 \ldots 3)$ act in spin and orbital space, respectively. To determine the coefficients $\alpha_{i}$ in equation (2), we performed a microscopic calculation (see Supplementary Methods) first including the $3 d_{x y}$ orbital together with the atomic spin-orbit coupling $\lambda \boldsymbol{L} \cdot \mathcal{S}$ and the hopping matrix elements $i \delta k_{y}\left(i \delta k_{x}\right)$ between the $3 d_{x y}$ and $3 d_{x z}$ $\left(3 d_{y z}\right)$ orbitals along the $y$ axis ( $x$ axis). The amplitude $\delta$, which can only be finite as the inversion symmetry is broken, has been shown in ref. 31, to be an essential ingredient for understanding the band structure of the interface electron system. Despite being shifted down in energy by $\epsilon_{0}$, we can project out the $3 d_{x y}$ band due to the spatial separation of the $3 d_{x y}$ and $3 d_{x z}\left(3 d_{y z}\right)$ orbitals. This yields the spin-orbit coupling in equation (2) with $\alpha_{0}=-\alpha_{1}=-\alpha_{3}=\frac{1}{2} \delta \lambda / \epsilon_{0}$. The values $\lambda \simeq 20 \mathrm{meV}$, $\delta / a_{0} \simeq 40 \mathrm{meV}$ with $a_{0}$ denoting the in-plane lattice constant, and $\epsilon_{0} \simeq 250 \mathrm{meV}$ were determined in first principles calculations $^{31}$. As $\delta$ and $\epsilon_{0}$ depend sensitively on details of the interface we use $\alpha_{0} \simeq 10 \ldots 50 \mathrm{meV} \AA$, estimated from magnetotransport experiments ${ }^{28}$.

In Fig. 1a the bands that result from the combination of the anisotropic masses in equation (1) and the spin-orbit coupling in equation (2) are shown. Two of the four bands are pushed to higher energies by the atomic spin-orbit coupling $\lambda \tau_{2} \sigma_{3}$ and can thus be neglected for the following low-energy analysis as long as the chemical potential is tuned sufficiently far away from the bottom of these bands. The remaining two bands are split by the Dresselhaus-Rashba coupling and show strong nesting in the highlighted regions. The nesting is a consequence of the mass anisotropy and becomes exact in the limit $m_{\mathrm{l}} / m_{\mathrm{h}} \rightarrow 0$.

This allows us to use a low-energy theory that involves only the degrees of freedom in the vicinity of the most parallel slices of the Fermi surface. In total, there are four equivalent strongly nested subspaces that are related by the point-group symmetries. Without loss of generality, let us focus on the one indicated in red in Fig. 1a. In this subset of momentum space, we introduce helicity annihilation and creation operators $c_{(\sigma, j)}$ and $c_{(\sigma, j)}^{\dagger}$ that diagonalize the quadratic part of the Hamiltonian. Here $\sigma= \pm$ refers to the sign of $k_{x}$ and $j=1(j=2)$ denotes the outer (inner) Fermi surface. To relate these operators to observables, Figs $1 b, c$ show the spin orientation and the orbital weight of the states in the vicinity of the outer and inner Fermi surface, respectively. We emphasize the similarity of both the form of the Fermi surface and the spin/orbital textures to the results reported in ref. 20 for the surface states of $\mathrm{SrTiO}_{3}$.

There are two types of interaction processes allowed by momentum conservation, which we will refer to as backscattering and forward scattering. The most general momentum-independent 

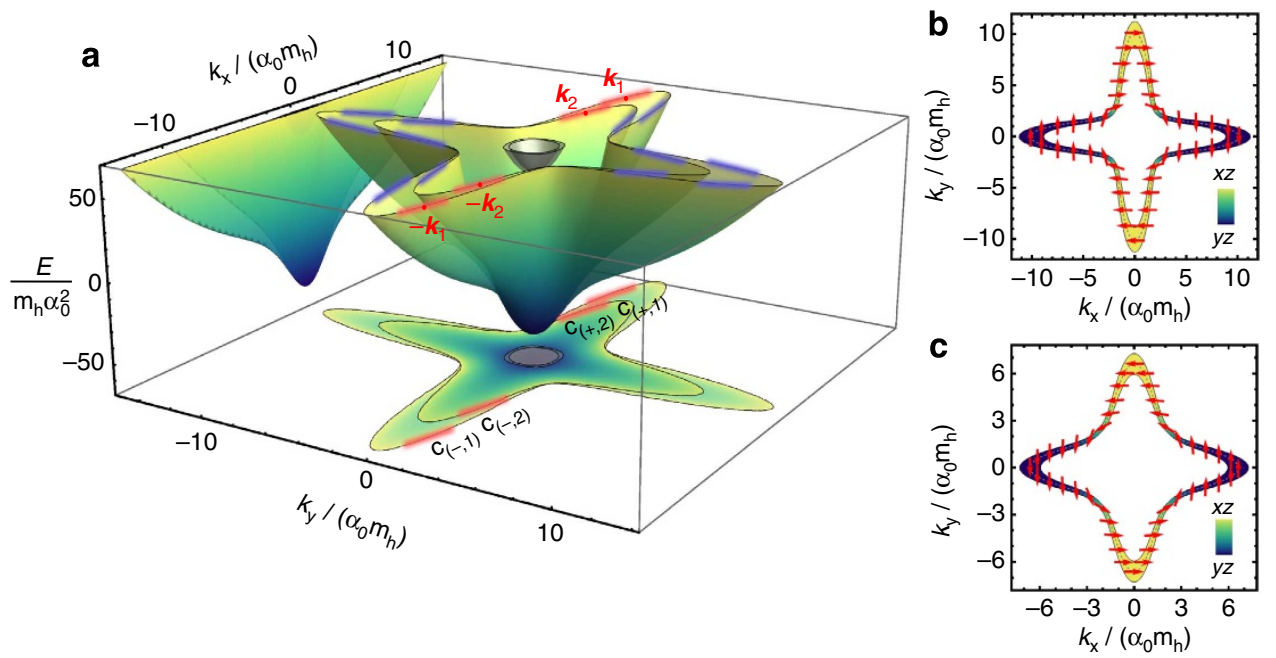

Figure 1 | The bands and the wave functions of our model. (a) The spectrum of the effective two-orbital Hamiltonian using the realistic parameters stated in the main text. In this paper, we restrict the analysis on the four most strongly nested subspaces (highlighted in red and blue). The orbital weight (colour) and orientation of the spin (red arrows) are illustrated in (b) and (c) for the outer and inner Fermi surface, respectively. Note that, as a consequence of time reversal and $\pi$-rotation symmetry about the $z$ axis, the spin has to lie in the $x y$ plane.

backscattering term is given by

$$
H_{\text {back }}=\sum_{s, s^{\prime}=0}^{3} \sum_{q} J_{s}^{-}(\boldsymbol{q}) u_{s s^{\prime}} J_{s^{\prime}}^{+}(-\boldsymbol{q})
$$

where $(\sigma= \pm)$

$$
J_{s}^{\sigma}(\boldsymbol{q})=\sum_{\boldsymbol{k}, j, j^{\prime}} c_{(\sigma, j)}^{\dagger}(\boldsymbol{k}+\boldsymbol{q})\left(\sigma_{s}\right)_{j, j^{\prime}} c_{\left(\sigma, j^{\prime}\right)}(\boldsymbol{k})
$$

From now on, the Pauli matrices $\sigma_{s}$, as in equation (4), do not describe the physical spin, but rather act in the abstract isospin space of the local helicity operators. The momentum of the operator $c_{(\sigma, j)}$ is measured relative to the centre $\sigma \boldsymbol{k}_{j}$ of the corresponding red region in Fig. 1a. Using the phase convention for the eigenstates defined in the Methods, one finds that the $\pi$ rotation symmetry with respect to the $z$ axis implies that $u$ has to be symmetric, $u^{T}=u$. The remaining symmetries of the point group then fully determine the interaction in the other three most strongly nested subspaces. In addition, time-reversal symmetry imposes the constraint $u_{s s^{\prime}}=0$ if either $s=2$ or $s^{\prime}=2$. Let us first assume that the cut-off $\Lambda_{\perp}$ for the momenta perpendicular to the Fermi surface can be chosen smaller than the distance between the inner and outer Fermi surface (below we will also discuss the opposite limit of nearly degenerate Fermi surfaces). This means that the red regions in Fig. 1a do not overlap. In this situation, momentum conservation rules out further interaction processes such that only $u_{00}, u_{11}=-u_{22}, u_{33}$ and $u_{30}=u_{03}$ can be nonzero. As the notation in equation (3) is rather abstract, we represent the four allowed backscattering terms in Figs 2a-d graphically in the helicity basis. One finds two distinct intraband Coulomb terms $\left(U_{1}, U_{2}\right)$, interband Coloumb interaction $\left(U^{\prime}\right)$ as well as pair hopping $(J)$.

In case of forward scattering, where all four fermions have the same index $\sigma$, the combination of Fermi statistics and point symmetries leads to only one independent coupling constant. The associated process $(V)$ is illustrated in Fig. 2 e.

Pairing instabilities. Having derived the interacting low-energy Hamiltonian, we can now deduce the associated instabilities. We perform a standard fermionic one-loop Wilson renormalization a

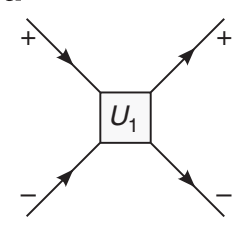

$u_{1}=u_{00}+u_{33}+2 u_{30}$ b

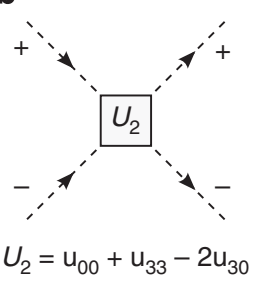

C

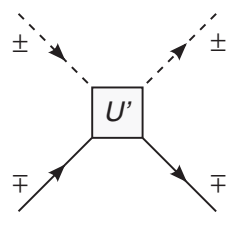

$U^{\prime}=u_{00}-u_{33}$ d

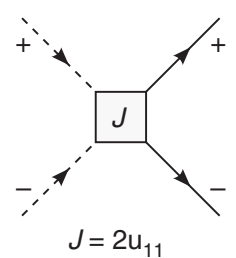

\section{e}

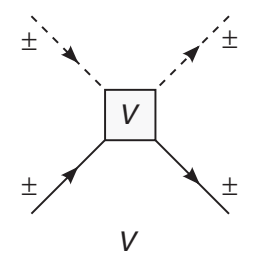

Figure 2 | Diagrammatic representation of the scattering processes. In case of strong spin-orbit coupling, the combination of momentum conservation, time reversal and rotation symmetry allow for four independent backscattering processes (a-d) and one forward scattering term (e). Solid and dashed lines refer to the outer and inner Fermi surface and \pm indicates the sign of $k_{x}$ in the four red regions of Fig. 1a.

group (RG) calculation ${ }^{32}$, in which high-energy degrees of freedom are successively integrated out yielding an effective Hamiltonian with renormalized coupling constants. If, during this procedure, some of the couplings diverge, the system will develop an instability. Following refs 33,34 we identify the physical nature of this instability by determining the order parameter that has the highest transition temperature, allowing for all possible (momentum independent) particle-hole and particle-particle ordered states:

$$
\begin{aligned}
\Delta_{\alpha, \beta}^{\mathrm{DW}} & :=\sum_{\boldsymbol{k}}\left\langle c_{\alpha}^{\dagger}(\boldsymbol{k}) c_{\beta}(\boldsymbol{k})\right\rangle, \\
\bar{\Delta}_{\alpha, \beta}^{\mathrm{SC}} & :=\sum_{\boldsymbol{k}}\left\langle c_{\alpha}^{\dagger}(\boldsymbol{k}) c_{\beta}^{\dagger}(-\boldsymbol{k})\right\rangle,
\end{aligned}
$$


where $\alpha$ and $\beta$ are double indices comprising helicity $\sigma= \pm$ and the Fermi surface sheet index $j=1,2$. Near the Fermi surface, we linearize the band dispersion $\epsilon(\boldsymbol{k}) \simeq \pm v_{j} k_{\perp}$ with $k_{\perp}$ denoting the component of the momentum perpendicular to the Fermi surface. For simplicity, let us first focus on the situation $v_{1}=v_{2}$, which is quantitatively a good approximation even when the chemical potential gets closer to the bottom of these bands. Below, we will also investigate the more general case $v_{1} \neq v_{2}$.

If $v_{1}=v_{2}$, only $u_{11}$ and $u_{33}$ out of the five coupling constants flow. In the associated flow diagram in Fig. 3a, we find two regimes, denoted by (I) and (II), where the running couplings diverge. Using the notation of Fig. 2 , it holds $-U_{1} \sim-U_{2} \sim$ $U^{\prime} \sim \pm J / 2 \gg|V|, U^{\prime}>0$, at the associated strong coupling fixed points with + and - referring to regime (I) and (II), respectively. In both cases, the instability is of superconducting type characterized by the two non-zero anomalous expectation values $\Delta_{(-, j),(+, j)}^{\mathrm{SC}}, j=1,2$, having equal modulus. As expected, there is only intra-Fermi surface pairing, implying that only Kramers partners are paired. In region (I), the superconducting order parameters of the nearby Fermi surfaces have opposite sign, whereas in (II) the sign is the same. The corresponding superconducting states will be denoted by $\mathrm{SC}^{+-}$and $\mathrm{SC}^{++}$, respectively. Rewriting the order parameters in the spin basis, one finds a mixture of singlet and triplet pairing, where both components are of equal strength in the strongly nested domains and the triplet pairing vector points perpendicular to the Fermi surface.

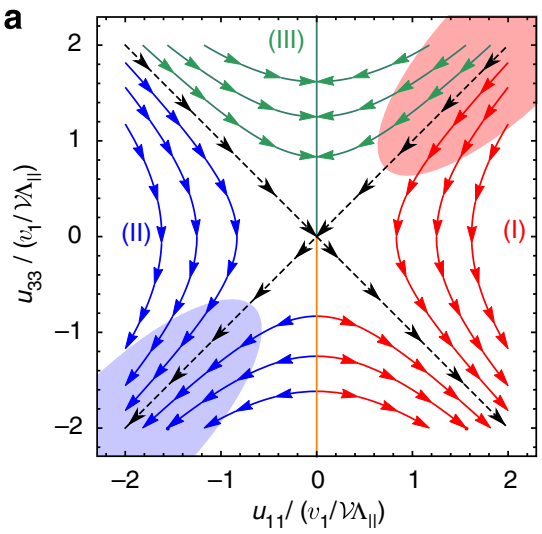

C

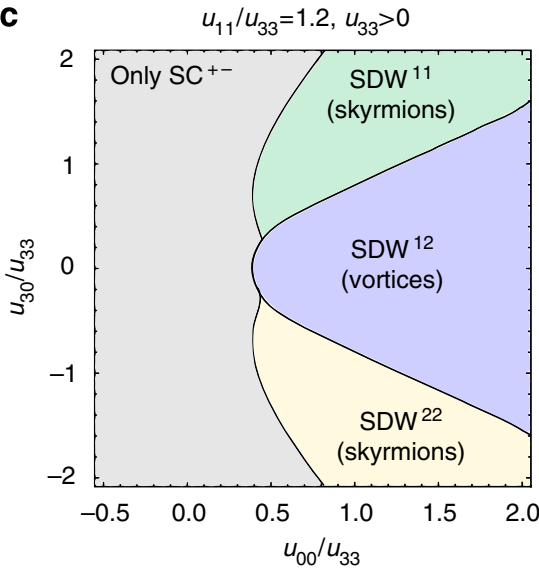

In the region (III), none of the coupling constants diverge, which means that for sufficiently small bare couplings the system will not develop any instability and, thus, reside in the metallic phase.

To unveil the microscopic pairing mechanism of the two superconducting states, we start from a repulsive Coulomb interaction between the $3 d$ orbitals and project onto the effective low-energy theory. This places us into region (I) of the RG flow in Fig. 3a. In contrast, an attractive interaction due to electron-phonon coupling would lead to initial couplings in region (II). Consequently, $\mathrm{SC}^{++}$results from conventional electron-phonon pairing, whereas $\mathrm{SC}^{+-}$is an unconventional superconductor, where particle-hole fluctuations effectively change the sign of $u_{33}$.

Topological superconductivity. Both $\mathrm{SC}^{+-}$and $\mathrm{SC}^{++}$respect time-reversal symmetry as far as the degrees of freedom of the nested subspaces are concerned. It is natural to assume that this holds for the entire Fermi surface and that, in addition, the system does not break the point symmetries relating the nested segments. In this case the gap is finite on the entire Fermi surface as seen in recent experiments ${ }^{35}$. Being fully gapped, it is obvious to ask whether the time-reversal invariant $2 \mathrm{D}$ superconductor (class $^{36}$ DIII) is topologically trivial or non-trivial ${ }^{37}$, which is of great interest as it strongly influences its physical properties. The most prominent feature of a non-trivial topological

b
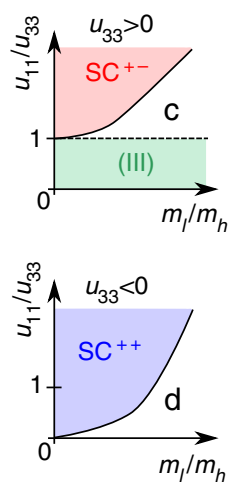

d

d $\quad u_{11} / u_{33}=0.8, u_{33}<0$

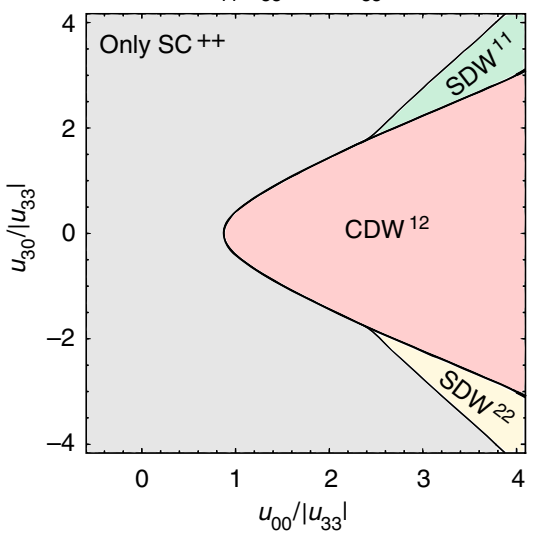

Figure 3 | RG flow and the resulting phase diagrams. (a) The flow of the two running coupling constants in the case of identical Fermi velocities is shown. Here $\mathcal{V}$ and $\Lambda_{\|}$denote the volume of the system and the cut-off of the nested subspaces tangential to the Fermi surface. Only in the regimes (I) and (II) the couplings diverge indicating that the system develops an instability. The red (blue) region corresponds to the bare couplings for a microscopically repulsive (attractive) interaction. The schematic phase diagrams taking into account finite mass anisotropies are shown in part (b). The non-flowing couplings determine the properties of the charge density wave as shown in (c) and (d) for the unconventional and conventional superconductor, respectively. The nomenclature of the phases is explained in the main text. 
superconductor is the appearance of spin-filtered counter propagating Majorana modes at its edge when surrounded by a trivial phase $\mathrm{s}^{38}$. It has been shown ${ }^{39}$ that, in the weak-pairing limit, the associated topological invariant $N \in \mathbb{Z}_{2}$ is fully determined by the sign of the paring field on the Fermi surfaces. For non-degenerate Fermi surfaces, it holds

$$
N=\prod_{j}\left(\operatorname{sign}\left(\delta_{j}\right)\right)^{m_{j}}, \quad \delta_{j}=\left\langle\psi_{j}\left|T \Delta_{j}^{\dagger}\right| \psi_{j}\right\rangle,
$$

where the product involves all Fermi surfaces, $\psi_{j}$ and $\Delta_{j}$ denote the wave function of the non-interacting part of the Hamiltonian and the pairing field at an arbitrary point on the jth Fermi surface. Furthermore, $m_{j}$ is the number of time-reversal invariant points enclosed by the $j$ th Fermi surface and $T$ is the unitary part of the time-reversal operator, given by $T=i \tau_{0} \sigma_{y}$ in the basis of equation (2). As, in the present case, both Fermi surfaces enclose only one time-reversal invariant point, the superconductor is topological (trivial) if the sign of $\delta_{j}$ is different (identical) on the two Fermi surfaces. Note that no additional Fermi surfaces are introduced when the continuum description of the theory is replaced by the properly lattice periodic tight-binding Hamiltonian. This is important as topological band theory is only well defined for lattice periodic systems.

Inserting the order parameters derived above, we obtain the pairing Hamiltonian

$$
\begin{aligned}
H_{\text {pair }} & =\Delta_{0} \sum_{\boldsymbol{k}, j, j^{\prime}} c_{(-, j)}(-\boldsymbol{k}) M_{j, j^{\prime}} c_{\left(+, j^{\prime}\right)}(\boldsymbol{k})+\text { H.c. }, \\
M & =\gamma_{0} \sigma_{0}+\gamma_{3} \sigma_{3},
\end{aligned}
$$

with $\gamma_{0}=u_{00}+2 u_{11}+u_{33}, \quad \gamma_{3}=2 u_{30}$ for the superconductor $\mathrm{SC}^{++}$and $\gamma_{0}=2 u_{30}, \gamma_{3}=u_{00}-2 u_{11}+u_{33}$ for the SC ${ }^{+-}$state. Calculating $\delta_{j}$ in equation (6), one finds (see Methods for more details) that the superconductor is topological if $\left|\gamma_{0}\right|<\left|\gamma_{3}\right|$ and trivial for the reversed inequality sign. At $\left|\gamma_{0}\right|=\left|\gamma_{3}\right|$, the gap closes as is characteristic for a topological phase transition. Recalling the flow depicted in Fig. 3a, one immediately sees that $\mathrm{SC}^{++}$is trivial, whereas $\mathrm{SC}^{+-}$is a topological superconductor. Accordingly, the experimental observation of topological features of the superconducting state implies that the pairing mechanism must be unconventional as it is the case for $\mathrm{SC}^{+-}$. Vice versa, a trivial state is only consistent with conventional, electron-phonon induced superconductivity.

Note that the pairing states obtained here differ from the results of former studies ${ }^{40-42}$ of interacting single-band models with Rashba spin-orbit coupling for the following reasons: The anisotropic masses lead to strong nesting, the inclusion of two orbitals allows both for more independent interaction terms and for additional spin-orbit couplings that crucially affect the spin textures.

Furthermore, we emphasize the difference of our result to recent work ${ }^{43-46}$ proposing the emergence of Majorana fermions in the heterostructure as a consequence of the coexistence of magnetism and superconductivity. In that case, however, (physical, spin-1/2) time-reversal symmetry is broken, whereas the $\mathrm{SC}^{+-}$state found here is time-reversal invariant.

Competing phases and spin textures. Eventually, our RG flow will always favour a superconducting state. However, by successively reducing the characteristic energy scale, we are increasingly sensitive to details of the low-energy theory and, consequently, the fact that the nesting is not perfect becomes relevant. For any finite $m_{1} / m_{\mathrm{h}}$, the strongly nested domains of the Fermi surface have a non-vanishing curvature, thus, eventually forcing the RG flow to stop. If the flow is terminated before any of the coupling constants diverges, other phases competing with superconductivity can emerge, as illustrated in Fig. 3b. In the associated mean-field equations, all coupling constants can now be of the same order and, hence, the resulting diagram of competing phases depends on all couplings, also on those that do not flow. The analysis yields that either a charge density wave $\left(\mathrm{CDW}^{12}\right)$ or three different spin density waves $\left(\mathrm{SDW}^{11}, \mathrm{SDW}^{22}\right.$, $\mathrm{SDW}^{12}$ ) are possible or the corresponding superconducting states are dominant for arbitrary $m_{\mathrm{l}} / m_{\mathrm{h}}$ as shown in Fig. $3 \mathrm{c}, \mathrm{d}$. The superscripts in the density waves $\mathrm{CDW}^{i j}$ and SDW ${ }^{i j}$ refer to the particle-hole expectation value $\Delta_{(-, i),(+, j)}^{\mathrm{DW}}$ (and $i \leftrightarrow j$, if $\left.i \neq j\right)$ that is non-zero in the respective phase. The difference between $\mathrm{CDW}^{12}$ and $\mathrm{SDW}^{12}$ is the relative sign of $\Delta_{(-, i),(+, j)}^{\mathrm{DW}}$ and $\Delta_{(-, j),(+, i)}^{\mathrm{DW}}$, rendering the order parameter symmetric and antisymmetric under time reversal in the former and in the latter case, respectively.

The spatial structure of the charge and spin density waves can easily be determined from the wave functions of the system and the order parameters $\Delta_{\alpha, \beta}^{\mathrm{DW}}$. In analogy to the discussion of the superconducting states, we assume that no additional point-group symmetry is broken. In the case of the $\mathrm{CDW}^{12}$ phase, one then finds that the local charge density is given by

$$
\rho(\boldsymbol{x}) \propto \cos \left(\boldsymbol{Q}_{12} \cdot \boldsymbol{x}\right)+\ldots,
$$

where $\boldsymbol{Q}_{12}=\boldsymbol{k}_{1}+\boldsymbol{k}_{2}$ is the associated nesting vector. The first contribution stems solely from the nested subspace highlighted in red in Fig. 1a and the ellipsis stands for the terms emanating from the remaining three subspaces, which are fully determined by the $\pi / 2$ rotation and reflection symmetry at the $x z$ plane. The resulting charge profile is illustrated in Fig. 4a. Note that the periodicity crucially depends on the ratio of the $x$ and $y$ component of the nesting vector $\boldsymbol{Q}_{12}$.

Similarly, the spatial structure of the spin density waves SDW $^{12}$ and SDW $^{11}, \mathrm{SDW}^{22}$ can be calculated (for details see Supplementary Methods) yielding the textures shown in Fig. $4 \mathrm{~b}$ and Fig. 4c, respectively. Here we have used that, in the red regions of Fig. 1a, the spins are approximately aligned along the $y$ axis (see Fig. 1b,c). Within this approximation, the expectation value of the spin lies in the $x y$ plane in case of the spin density phase SDW ${ }^{12}$. The $2 \mathrm{D}$ vector field is a lattice of vortices, both with positive and negative winding numbers. In the phases $\mathrm{SDW}^{11}$ and $\mathrm{SDW}^{22}$, the spin is free to rotate in three dimensions. One finds a complicated periodic arrangement of isolated skyrmions and antiskyrmions, as well as closely bound skyrmion-antiskyrmion pairs (see Fig. 4d). The emergence of a skyrmion lattice that leads to interesting physical effects (see for example ref. 47), is consistent with recent work ${ }^{8,48}$ pointing out that these magnetic topological defects naturally appear as solutions of the Ginzburg-Landau equations for systems with spin-orbit interaction.

On top of that, the difference between the density wave phases in Figs 3c,d neighbouring the superconducting states $\mathrm{SC}^{+-}$and $\mathrm{SC}^{++}$can be exploited to gain information about the pairing mechanism in the heterostructure. As the orbital contribution to the magnetization is negligible for large mass anisotropies, the experimental observation of in-plane magnetization ${ }^{5}$ is only consistent with the $\mathrm{SDW}^{12}$ state. This implies that the superconducting phase of $\mathrm{SrTiO}_{3} / \mathrm{LaAlO}_{3}$ is supposed to be unconventional and topologically non-trivial.

\section{Discussion}

As the $\mathbb{Z}_{2}$ invariant $N$ is a rather abstract way of classifying superconducting phases, it is worthwhile to gain intuitive understanding for why identical (different) signs of the pairing fields lead to a topologically trivial (non-trivial) superconductor. Imagine starting from the standard $s$-wave singlet BCS 

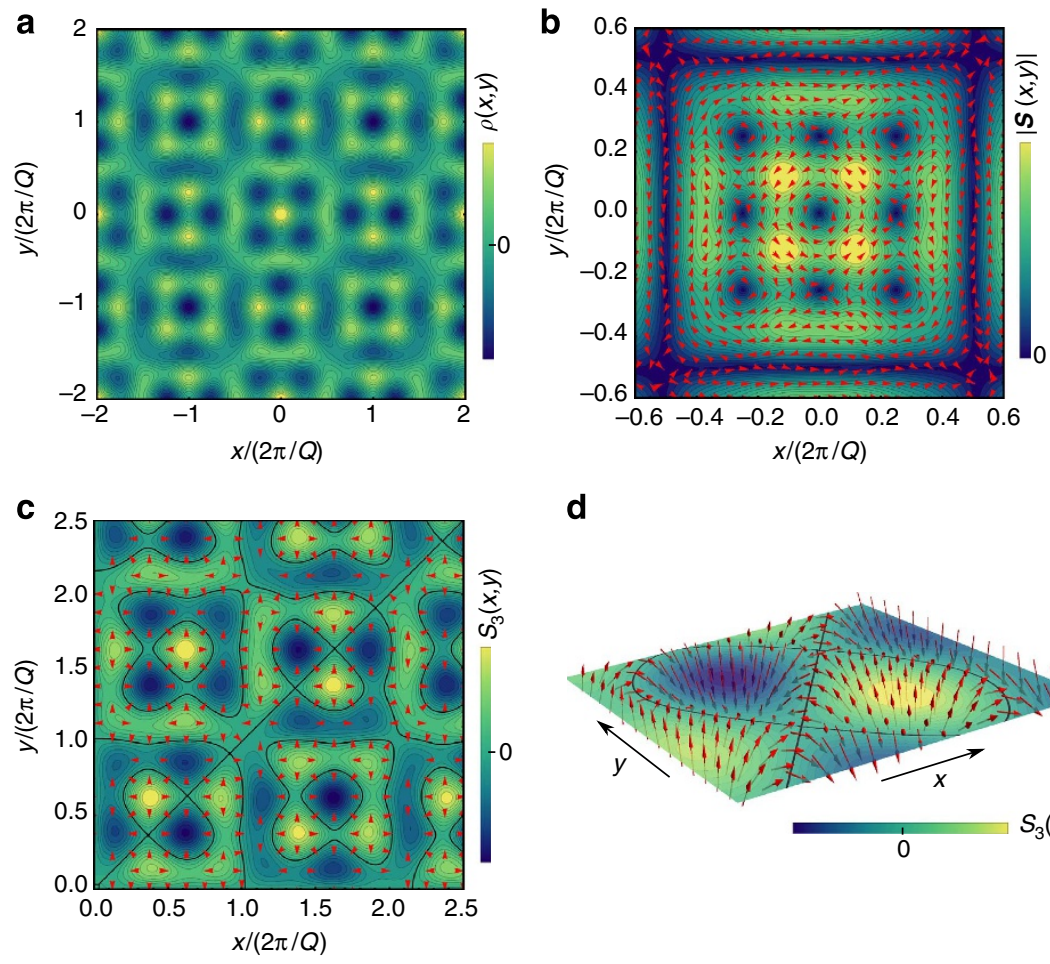

d

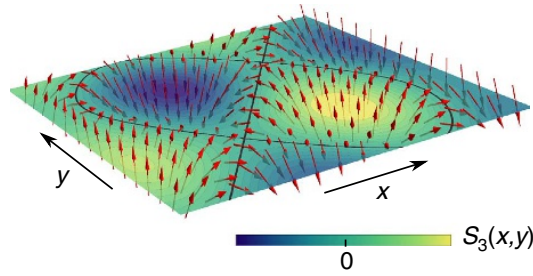

Figure 4 | The spatial structure of the different density wave phases. (a) The charge density pattern of CDW ${ }^{12}$. In SDW ${ }^{12}$, where the nesting vector is again given by $\mathbf{Q}_{12}=\boldsymbol{k}_{1}+\boldsymbol{k}_{2}$, the spin $\mathcal{S}$ lies approximately in the xy plane. As shown in (b) using red arrows to indicate the direction of the spin, one finds a lattice of vortices. In case of SDW ${ }^{11}$, the nesting vector is $2 \mathbf{k}_{1}$ and we observe a lattice of skyrmions and antiskyrmions as illustrated in (c), where the red arrows indicate the direction of the $x y$ components of the spin and the black lines are the zeros of its $z$ component $\mathcal{S}_{3}$. (d) One of the emerging closely bound skyrmion-antiskyrmion pairs. The texture of SDW ${ }^{22}$ (nesting vector $2 \boldsymbol{k}_{2}$ ) is identical to SDW ${ }^{11}$ up on replacing $\mathcal{S}_{3} \rightarrow-\mathcal{S}_{3}$. In all plots, a nesting vector of the form $(2,0.48) Q$ has been assumed.

superconductor, characterized by an isotropic, spin-degenerate Fermi surface, where only Kramers partners are paired. Turning on terms that break inversion and rotation symmetry, this phase can be continuously deformed into the $\mathrm{SC}^{++}$state without closing the gap as illustrated in Fig. 5. This proves that the $\mathrm{SC}^{++}$ phase is, exactly as the BCS superconductor, topologically trivial. Importantly, relative phases between the pairing fields of the two Fermi surfaces cannot be introduced adiabatically without breaking time-reversal symmetry. Therefore, deforming the BCS state continuously into the $\mathrm{SC}^{+-}$superconductor necessarily involves a closing of the gap on one of the Fermi surfaces. At this gap closing, a topological phase transition occurs as we have shown mathematically by calculating the invariant $N$ for the $\mathrm{SC}^{+-}$state. Note that a similar topological phase has been found very recently in a prototype model of $2 \mathrm{D}{ }^{3} \mathrm{He}-\mathrm{B}$ with the assumption of Rashba spin-orbit coupling ${ }^{49}$.

Our link between topology and pairing mechanism allows to unveil the unknown microscopic physics of the superconductor by observing signatures of Majorana bound states: In case of the unconventional $\mathrm{SC}^{+-}$state, counter propagating Majorana modes are expected to be localized on a length scale of the order of the coherence length ${ }^{2} \xi \simeq 100 \mathrm{~nm}$ at boundaries to trivially gapped phases. These modes can be probed experimentally, for example, using tunnel spectroscopy or via thermal conductivity measurement ${ }^{50}$. From the material science point of view, it is important to distinguish between 'intrinsic' and 'extrinsic' topological superconductors. In 'extrinsic' systems (see, for example, refs 16-18) Cooper pairs are injected via the proximity effect from a non-topological superconductor into another conductor that is thereby driven into a topological superconducting phase. The $\mathrm{SC}^{+-}$state is an 'intrinsic'

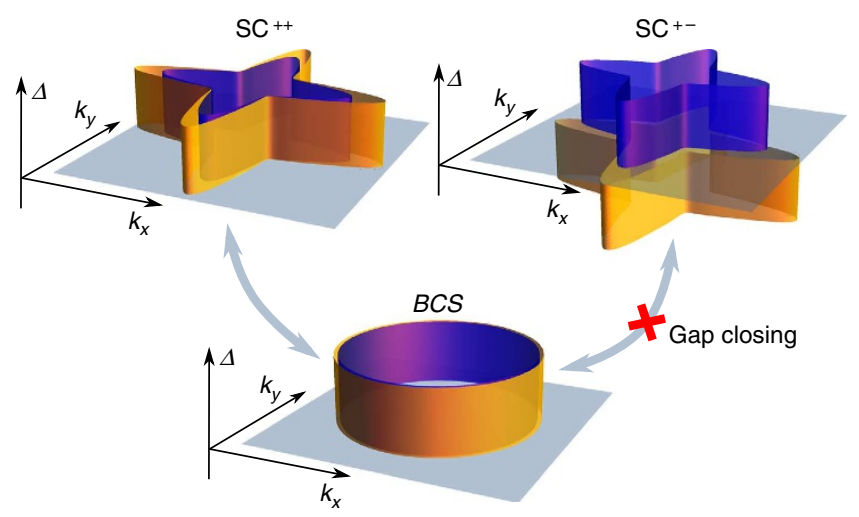

Figure 5 | Illustration of the topological properties. The value of the pairing field at the inner (blue) and outer (orange) Fermi surface is shown for the $s$-wave singlet BCS superconductor as well as for the $\mathrm{SC}^{++} / \mathrm{SC}^{+-}$ phases. The topologically trivial BCS state is adiabatically connected to the $\mathrm{SC}^{++}$superconductor, whereas a continuous deformation into the $\mathrm{SC}^{+-}$ phase requires closing the gap at some point along the path.

topological superconductor, where the pairing and the non-trivial topology emerges from the material's internal dynamics, which could simplify probing Majorana physics. One additional advantage of oxide interfaces for investigating Majorana modes is that boundaries of highly controllable shape between superconducting and insulating phases can be realized experimentally, either via locally reducing the thickness of the $\mathrm{LaAlO}_{3}$ crystal ${ }^{51}$ or using a voltage-biased atomic force microscope tip ${ }^{52}$. 
As already stated above, we have also considered the case of different Fermi velocities, $v_{1} \neq v_{2}$ (see Supplementary Note 1 for more details of the analysis). Then all four backscattering couplings, the interaction terms in Figs $2 \mathrm{a}-\mathrm{d}$, flow. Nonetheless, exactly as before, the leading instability is generically superconducting for sufficiently large mass anisotropies. Detuning the Fermi velocities leads to a crossover from the superconductors $\mathrm{SC}^{+-}$and $\mathrm{SC}^{++}$, which are characterized by $\left|\Delta_{(-, 1),(+, 1)}^{\mathrm{SC}}\right|=\left|\Delta_{(-, 2),(+, 2)}^{\mathrm{SC}}\right|$, to a pairing state where the anomalous expectation value $\Delta_{(-, j),(+, j)}^{\mathrm{SC}}$ is only finite on the Fermi surface with the larger Fermi velocity. Note that, in this limit, the associated mean-field Hamiltonian still describes pairing on both Fermi surfaces due to the pair hopping term, giving rise to a fully gapped phase. Remarkably, we again find that the superconductor resulting from the conventional electronphonon pairing mechanism is topologically trivial, whereas the unconventional superconductor is non-trivial. This proves that the correspondence between the pairing mechanism and the topological properties of the superconducting phases in the heterostructure holds irrespective of the values of the Fermi velocities.

For completeness, we have also investigated the case of very weak spin-orbit interaction where the energetic cut-off of the low-energy model is much larger than the spin-orbit splitting (see Supplementary Note 2). Then the red regions in Fig. 1a merge pairwise and, consequently, momentum conservation is much less restrictive making more backscattering terms possible. Surprisingly, even in this situation, the observation of a topologically non-trivial superconducting phase is only consistent with the pairing mechanism being unconventional.

Finally, we point out that the pairing state of a many-body system is always a consequence of the combination of electronphonon and electron-electron interactions. Even if phonons enhance a sign-changing pairing state, the electron-phonon coupling alone prefers quite generally conventional pairing (see, for example, refs 53-55). This includes the recently proposed scenario, where odd-parity pairing states and related topological superconductivity ${ }^{13}$ are predicted to occur in systems with an electron-phonon coupling that is singular at long wavelength and closely tied to inversion symmetry ${ }^{56,57}$. The interesting mechanism of refs 56,57 does not apply to the oxide interfaces studied here, as inversion symmetry is broken, the transferred momentum is finite, and since tunnelling data indicate a fully established superconducting gap ${ }^{35}$. Thus, we conclude that for the oxide interfaces the established link between topology and microscopic mechanism is robust.

In summary, our analysis reveals that the phase diagram of the $2 \mathrm{D}$ electron fluid forming at the interface between the perovskite oxides $\mathrm{LaAlO}_{3}$ and $\mathrm{SrTiO}_{3}$ combines two fascinating notions of condensed matter physics: Topology and unconventional superconductivity. We find that, very generically, the observation of signatures of topologically non-trivial superconductivity, such as the appearance of Majorana bond states, directly implies that the underlying pairing mechanism must be unconventional. In addition, the spin density wave phases competing with topological superconductivity show topological spatial textures as well. Depending on the value of the coupling constants, we find lattices of both skyrmions and vortices.

\section{Methods}

Phase convention for the helicity states. Using a path-integral representation, the quadratic part of the theory can be written as

$$
S_{0}=T \sum_{\omega_{n}} \sum_{\boldsymbol{k}} \bar{\Psi}_{\alpha}(k)\left[-i \omega_{n} \delta_{\alpha, \beta}+H_{\alpha, \beta}(\boldsymbol{k})\right] \Psi_{\beta}(k),
$$

where $k \equiv\left(\omega_{n}, \boldsymbol{k}\right)$ and $\Psi, \bar{\Psi}$ are four-component Grassmann fields describing spinful fermions in the $3 d_{x z}$ and $3 d_{y z}$ orbitals. Furthermore, $H$ is the Hamiltonian defined in the main text characterized by the anisotropic masses (1) and the spinorbit coupling in equation (2).

We diagonalize $S_{0}$ by performing the unitary transformation

$$
\Psi_{\alpha}(k)=\mathcal{U}_{\alpha, \alpha^{\prime}}(\boldsymbol{k}) f_{\alpha^{\prime}}(k), \quad \bar{\Psi}_{\alpha}(k)=\mathcal{U}_{\alpha, \alpha^{\prime}}^{*}(\boldsymbol{k}) \bar{f}_{\alpha^{\prime}}(k),
$$

where

$$
\mathcal{U}(\boldsymbol{k})=\left[\phi_{1}(\boldsymbol{k}), \phi_{2}(\boldsymbol{k}), \phi_{3}(\boldsymbol{k}), \phi_{4}(\boldsymbol{k})\right]
$$

with $\phi_{\alpha}(\boldsymbol{k})$ denoting an eigenvector of $H(\boldsymbol{k})$. As explained in the main text, we can restrict the analysis of instabilities to one of the most strongly nested subspaces and introduce helicity fields $c\left(_{\sigma, j}\right)$ and $\bar{c}_{(\sigma, j)}$ in the local coordinate systems yielding

$$
S_{0}=\int_{k} \bar{c}_{(\sigma, j)}(k)\left[-i \omega_{n}+\sigma v_{j} k_{\perp}+s_{j} \eta\right] c_{(\sigma, j)}(k)
$$

after linearizing the spectrum. Here $s_{1}=+1, s_{2}=-1$ and $\eta$ denotes the spin-orbit splitting in the case of quasi-degenerate Fermi surfaces. For stronger spin-orbit coupling, where the four red regions in Fig. 1a are disjoint, one has $\eta=0$ by construction. The different regimes of the quasi one-dimensional description in equation (12) are summarized graphically in Supplementary Fig. 1. In equation (12) and in the following, we use the compact notation $k \equiv\left(\omega_{n}, k_{\|}, k_{\perp}\right)$ and

$$
\int_{k} \cdots=T \sum_{\omega_{n}} \int_{-\Lambda_{\|}}^{\Lambda_{\|}} \frac{\mathrm{d} k_{\|}}{2 \pi} \int_{-\Lambda_{\perp}}^{\Lambda_{\perp}} \frac{\mathrm{d} k_{\perp}}{2 \pi} \ldots,
$$

where $\Lambda_{\perp}$ and $\Lambda_{\|}$are the momentum cutoffs normal and tangential to the Fermi surface.

To make the helicity operators unique, we have to fix the phases of the eigenstates in equation (11). This is achieved by exploiting the invariance of the Hamiltonian under $\pi$-rotation $R^{c_{2}}$ and time-reversal $\Theta$. The former symmetry implies that

$$
H(\boldsymbol{k})=\mathcal{R}_{\Psi}^{c_{2}} H(-\boldsymbol{k}) \mathcal{R}_{\Psi}^{c_{2} \dagger}, \quad \mathcal{R}_{\Psi}^{c_{2}}=i \tau_{0} \sigma_{3},
$$

and hence we can construct the eigenstates with negative $k_{x}$ from those with $k_{x}>0$ via

$$
\phi_{\alpha}(-\boldsymbol{k}):=\mathcal{R}_{\Psi}^{c_{2}^{\dagger}} \phi_{\alpha}(\boldsymbol{k}), \quad k_{x}>0 .
$$

Consecutive application of time reversal and $\pi$-rotation leads to the $\boldsymbol{k}$-space local antiunitary symmetry

$$
H(\boldsymbol{k})=\tau_{0} \sigma_{1} H^{*}(\boldsymbol{k}) \tau_{0} \sigma_{1}
$$

of the Hamiltonian. Thus, we can adjust the phases of the eigenstates such that

$$
\phi_{\alpha}(\boldsymbol{k})=\tau_{0} \sigma_{1} \phi_{\alpha}^{*}(\boldsymbol{k})
$$

for $k_{x}>0$. From equation (15), it follows that equation (17) actually holds also for $k_{x}<0$.

Representation of the symmetries. Having fixed the phases of the local eigenstates, the representation of time-reversal and $\pi$-rotation symmetry on the helicity fields is well defined. Note that the remaining elements of the point-group $C_{4 v}$ cannot be represented on $c$ and $\bar{c}$ as these symmetry operations act between different most strongly nested subspaces. For the very same reason, however, these symmetries are also irrelevant when deriving the most general interaction within one of the subspaces.

Time reversal acts according to

$$
\begin{aligned}
& \Psi_{\alpha}(\omega, \boldsymbol{k}) \stackrel{\Theta}{\longrightarrow}\left(i \sigma_{2}\right)_{\alpha, \beta} \bar{\Psi}_{\beta}(\omega,-\boldsymbol{k}), \\
& \bar{\Psi}_{\alpha}(\omega, \boldsymbol{k}) \stackrel{\Theta}{\longrightarrow} \Psi_{\beta}(\omega,-\boldsymbol{k})\left(i \sigma_{2}\right)_{\beta, \alpha}
\end{aligned}
$$

in the basis of equation (9). Using equations (15) and (17), one can derive the transformation behaviour

$$
\begin{aligned}
& c_{( \pm, j)}\left(\omega, k_{\|}, k_{\perp}\right) \stackrel{\Theta}{\longrightarrow} \mp i \bar{c}_{(\mp, j)}\left(\omega,-k_{\|},-k_{\perp}\right), \\
& \bar{c}_{( \pm, j)}\left(\omega, k_{\|}, k_{\perp}\right) \stackrel{\Theta}{\longrightarrow} \mp i c_{(\mp, j)}\left(\omega,-k_{\|},-k_{\perp}\right)
\end{aligned}
$$

in the helicity basis. Similarly, for the $\pi$-rotation symmetry, we find

$$
c_{( \pm, j)}\left(\omega, k_{\|}, k_{\perp}\right) \stackrel{R^{c_{2}}}{\longrightarrow} \mp c_{(\mp, j)}\left(\omega,-k_{\|},-k_{\perp}\right)
$$

and the same for $\bar{c}$.

Symmetry analysis of the interaction. Now we will derive the most general momentum-independent interaction of the low-energy theory consistent with the 
symmetries of the system. Let us write

$$
S_{\text {int }}=\int_{k_{1}, k_{2}, k_{3}, k_{4}} \bar{c}_{\alpha}\left(k_{4}\right) \bar{c}_{\beta}\left(k_{3}\right) c_{\gamma}\left(k_{2}\right) c_{\delta}\left(k_{1}\right) \mathcal{W}_{\gamma \delta}^{\alpha \beta} \delta\left(k_{1}+k_{2}-k_{3}-k_{4}\right)
$$

where the Greek letters are double indices comprising $\sigma= \pm$ and $j=1,2$. It turns out that the dimensionless parameterization,

$$
\omega_{\gamma \delta}^{\alpha \beta}=\frac{\Lambda_{\|}}{2 \pi^{2} v_{1}} \mathcal{W}_{\gamma \delta}^{\alpha \beta}
$$

with

$$
\omega_{\left(\sigma_{\gamma}, j_{\gamma}\right)\left(\sigma_{\delta}, j_{\delta}\right)}^{\left(\sigma_{\alpha}, j_{\alpha}\right)\left(\sigma_{\beta}, j_{\beta}\right)}= \begin{cases}V_{j_{\gamma}, j_{\delta}}^{j_{\alpha}, j_{\beta}}(\sigma), & \sigma_{\alpha}=\sigma_{\beta}=\sigma_{\gamma}=\sigma_{\delta}=\sigma, \\ W_{j_{\gamma}, j_{\delta}}^{j_{\alpha}, j_{\beta}}, & \left(\sigma_{\alpha}, \sigma_{\beta}, \sigma_{\gamma}, \sigma_{\delta}\right)=(-,+,+,-), \\ W_{j_{\delta}, j_{\gamma}}^{j_{\beta}, j_{\alpha}}, & \left(\sigma_{\alpha}, \sigma_{\beta}, \sigma_{\gamma}, \sigma_{\delta}\right)=(+,-,,+), \\ -W_{j_{\gamma}, j_{\delta}}^{j_{\beta}, j_{\alpha}}, & \left(\sigma_{\alpha}, \sigma_{\beta}, \sigma_{\gamma}, \sigma_{\delta}\right)=(+,-,+,-), \\ -W_{j_{\delta}, j_{\gamma}}^{j_{\alpha}, j_{\beta}}, & \left(\sigma_{\alpha}, \sigma_{\beta}, \sigma_{\gamma}, \sigma_{\delta}\right)=(-,+,-,+), \\ 0, & \text { otherwise, }\end{cases}
$$

is very convenient for the following analysis. In equation (23), we have already taken into account that only forward scattering (described by $V$ ) and backscattering $(W)$ are allowed by momentum conservation. Throughout this work, we assume that Umklapp processes are not possible. Due to Fermi statistics, the forward scattering tensors must have the form

$$
V_{j_{y}, j_{\delta}}^{j_{\alpha}, j_{\beta}}(\sigma)=g_{0}(\sigma)\left[\delta_{j_{\alpha}, j_{\delta}} \delta_{j_{\beta}, j_{\gamma}}-\delta_{j_{\alpha}, j_{\gamma}} \delta_{j_{\beta}, j_{\delta}}\right],
$$

whereas the backscattering tensor has 16 degrees of freedom, which we parametrize according to

$$
W_{j_{\gamma}, j_{\delta}}^{j_{\alpha}, j_{\beta}}=\sum_{s, s^{\prime}=0}^{3} g_{s s^{\prime}}\left(\sigma_{s}\right)_{j_{\alpha}, j_{\delta}}\left(\sigma_{s^{\prime}}\right)_{j_{\beta}, j_{\gamma}} .
$$

Note that $g_{s s^{\prime}} \propto u_{s s^{\prime}}$ with $u_{s s^{\prime}}$ used in the main text to define the backscattering terms.

Next, let us derive the constraints resulting from $\pi$-rotation symmetry. Demanding that equation (21) be invariant under the transformation (20), we find

$$
\begin{gathered}
V_{j_{\gamma}, j_{\delta}}^{j_{\alpha}, j_{\beta}}(+) \stackrel{!}{=} V_{j_{\gamma}, j_{j}}^{j_{\alpha}, j_{\beta}}(-), \\
W_{j_{\delta}, j_{\gamma}}^{j_{\beta}, j_{\alpha}} \stackrel{!}{=} W_{j_{\gamma}, j_{\delta}}^{j_{\alpha}, j_{\beta}} .
\end{gathered}
$$

Consequently, all forward scattering processes are characterized by one coupling constant $g_{0} \equiv g_{0}(+)=g_{0}(-)$. In terms of the representation in equation (25), the second constraint is equivalent to $g^{T}=g$, as stated in the main text.

Similarly, to make the interaction time-reversal symmetric, we require invariance of equation (21) under equations (19a,b). Again using the parameterization in equation (23), we find that $V$ is not further restricted, whereas the backscattering tensor has to satisfy

$$
W_{j_{\beta}, j_{\alpha}}^{j_{\delta}, j_{\gamma}} \stackrel{!}{=} W_{j_{y}, j_{\delta}}^{j_{\alpha}, j_{\beta}} .
$$

In the equation (25), this is equivalent to demanding $g_{s s^{\prime}}=0$ if either $s=2$ or $s^{\prime}=2$ and, hence, we have

$$
g=\left(\begin{array}{cccc}
g_{00} & g_{10} & 0 & g_{30} \\
g_{10} & g_{11} & 0 & g_{31} \\
0 & 0 & g_{22} & 0 \\
g_{30} & g_{31} & 0 & g_{33}
\end{array}\right)
$$

If the most strongly nested subspaces are disjoint, momentum conservation rules out further interaction terms leading to the four independent backscattering couplings discussed in the main text.

Microscopic interaction. An important part of our analysis is the identification of the pairing mechanisms in the different superconductors. For this purpose, we include matrix elements of the electron-electron interaction between the relevant $3 d_{x y}$ and $3 d_{y z}$ orbitals yielding both an intra- $(\mathcal{U})$ and inter-orbital $\left(\mathcal{U}^{\prime}\right)$ Hubbard interaction, a Hund's coupling $\left(\mathcal{J}_{H}\right)$ term, as well as pair hopping $(\mathcal{J})$. In addition, we use $\mathcal{J}_{H}=\mathcal{J}$ and $\mathcal{U}=\mathcal{U}^{\prime}+2 \mathcal{J}$ valid for the usual Coulomb interaction, but our results do not crucially depend on this assumption.

Projecting the interaction onto the low-energy theory, we find, using the model defined in the main text,

$$
g_{00} \simeq g_{11} \simeq g_{33}, \quad\left|g_{00}\right| \gg\left|g_{30}\right|
$$

in case of disjoint support in momentum space. In this way, we can estimate the initial conditions for the RG flow both for a microscopically repulsive $\left(g_{00}>0\right)$ and for an electron-phonon induced, attractive $\left(g_{00}<0\right)$ interaction. The two scenarios correspond, respectively, to the red- and blue-shaded regions in Fig. 3a.
Wilson RG. In the Wilson approach, as discussed in detail for fermions with a finite Fermi surface in ref. 32, fast modes with momenta $\Lambda_{\perp} e^{-\Delta l}<k_{\perp}<\Lambda_{\perp}, \Delta l$ $>0$, are integrated out yielding, after proper rescaling, an effective action with renormalized parameters.

At one-loop order and for $T=0$, we find that, in all three regimes considered in this work, forward scattering is not renormalized, whereas the backscattering tensor flows according to

$$
\frac{\mathrm{d} W_{j_{y}, j_{\delta}}^{j_{\alpha}, j_{\beta}}}{\mathrm{d} l}=\sum_{j_{\mu}, j_{v}} \frac{4}{x_{j_{\mu}}+x_{j_{v}}}\left(W_{j_{\gamma}, j_{\mu}}^{j_{\alpha}, j_{v}} W_{j_{v}, j_{\delta}}^{j_{\mu}, j_{\beta}}-W_{j_{v}, j_{\mu}}^{j_{\alpha}, j_{\beta}} W_{j_{y}, j_{\delta}}^{j_{\mu}, j_{v}}\right)
$$

with $x_{j}:=v_{j} / v_{1}$. In the simplest case of strong spin-orbit coupling and $v_{1}=v_{2}$, equation (30) is equivalent to

$$
\frac{\mathrm{d} g_{11}}{\mathrm{~d} l}=-8 g_{11} g_{33}, \quad \frac{\mathrm{d} g_{33}}{\mathrm{~d} l}=-8 g_{11}^{2},
$$

yielding the flow shown in Fig. 3a.

Mean-field equations and instabilities. As we are only interested in determining the instability with the highest transition temperature, it is justified to linearize the mean-field equations in the order parameters. Keeping only the leading terms in the physically relevant limit $T \ll v_{2} \Lambda_{\perp}$, we find

$$
\Delta_{\alpha, \beta}^{\mathrm{DW}} \sim L_{j_{\alpha}, j_{\beta}}\left[\delta_{\sigma_{\alpha},-} \delta_{\sigma_{\beta},+} W_{j_{\beta}^{\prime}, j_{\alpha}}^{j_{\alpha}^{\prime}, j_{\beta}} \Delta_{\left(-, j_{\alpha}^{\prime}\right)\left(+j_{\beta}^{\prime}\right)}^{\mathrm{DW}}+\delta_{\sigma_{\alpha},+} \delta_{\sigma_{\beta},-} W_{j_{\alpha}, j_{\beta}^{\prime}}^{j_{\beta}, j_{\alpha}^{\prime}} \Delta_{\left(+, j_{\alpha}^{\prime}\right)\left(-j_{\beta}^{\prime}\right)}^{\mathrm{DW}}\right]
$$

and

$$
\bar{\Delta}_{\left(-, j_{\alpha}\right)\left(+, j_{\beta}\right)}^{\mathrm{SC}} \sim-2 L_{j_{\alpha}, j_{\beta}} W_{j_{\beta}, j_{\alpha}}^{j_{\alpha}^{\prime}, j_{\beta}^{\prime}} \bar{\Delta}_{\left(-, j_{\alpha}^{\prime}\right)\left(+, j_{\beta}^{\prime}\right)}^{\mathrm{SC}},
$$

where

$$
L_{j_{\alpha}, j_{\beta}}:=\frac{\left[\log \left(\frac{v_{1} \Lambda_{\perp}}{T}\right)\right]^{x_{j \alpha}}+\left[\log \left(\frac{v_{1} \Lambda_{\perp}}{T}\right)\right]^{x_{j \beta}}}{x_{j_{\alpha}}+x_{j_{\beta}}}
$$

has been introduced. In equation (32b), it has been exploited that $\bar{\Delta}_{\alpha, \beta}^{\mathrm{SC}}$ is antisymmetric such that it is sufficient to consider $\left(\sigma_{\alpha}, \sigma_{\beta}\right)=(-,+)$. We see that, both for the density wave and for the superconducting channel, solely order parameters with $\sigma_{\alpha}=-\sigma_{\beta}$ are relevant. In addition, only the backscattering tensor $W$ enters, whereas forward scattering, $V$, does not have any role at all.

Expanding the density wave order parameters,

$$
\Delta_{\alpha, \beta}^{\mathrm{DW}}=\sum_{s, s^{\prime}=0}^{3} c_{s, s^{\prime}}\left(\tau_{s}\right)_{\sigma_{\alpha}, \sigma_{\beta}}\left(\sigma_{s^{\prime}}\right)_{j_{\alpha}, j_{\beta}}, \quad c_{s, s^{\prime}} \in \mathbb{R}
$$

and the anomalous expectation values,

$$
\bar{\Delta}_{\left(-, j_{\alpha}\right),\left(+, j_{\beta}\right)}^{\mathrm{SC}}=\sum_{s=0}^{3} \tilde{c}_{s}\left(\sigma_{s}\right)_{j_{\alpha}, j_{\beta}}, \quad \tilde{\mathcal{c}}_{s} \in \mathbb{C},
$$

in Pauli matrices we can rewrite equations (32a,b) more explicitly. The result for strong spin-orbit coupling and identical Fermi velocities is summarized in Supplementary Table 1 . Inserting the asymptotic behaviour of the coupling constants, that is $g_{11} \sim-g_{33} \rightarrow \infty$ and $g_{11} \sim g_{33} \rightarrow-\infty$ in regime (I) and (II), respectively, one finds the superconducting phases $\mathrm{SC}^{+-}$and $\mathrm{SC}^{++}$discussed in the main text. To derive the competing instabilities, we have investigated the flow of all mean-field equations in Supplementary Table 1 according to equation (31) and analysed which of the order parameters is dominant before superconductivity eventually wins.

Calculation of the topological invariant. To obtain $N$ in equation (6), we have to relate the effective one-dimensional theory, equations (7) and (12), to the full mean-field Hamiltonian,

$$
\begin{aligned}
H_{\mathrm{MF}}^{2 \mathrm{D}}= & \sum_{\boldsymbol{k}} \Psi_{\alpha}^{\dagger}(\boldsymbol{k}) H_{\alpha, \beta}(\boldsymbol{k}) \Psi_{\beta}(\boldsymbol{k}) \\
& +\frac{1}{2} \sum_{\boldsymbol{k}}\left[\Psi_{\alpha}(-\boldsymbol{k}) \Delta_{\alpha, \beta}^{\dagger}(\boldsymbol{k}) \Psi_{\beta}(\boldsymbol{k})+\text { H.c. }\right],
\end{aligned}
$$

defined on the entire 2D Brillouin zone. For simplicity, let us focus on the case of strong spin-orbit coupling and $v_{1}=v_{2}$ (see Supplementary Notes 1 and 2 for the other regimes of the system). Suppose that the free Hamiltonian $H$ has been diagonalized by applying the transformation in equation (10). Here we use the convention that the eigenfunctions $\phi_{\alpha}$ are sorted for every $\boldsymbol{k}$ such that the energy increases with $\alpha$. From equation (7), we know that the pairing term, that is the second line in equation (36), must have the form

$$
H_{\text {pair }}^{2 \mathrm{D}}=\Delta_{0} \sum_{j=1,2} \sum_{\boldsymbol{k} \in S_{j}} f_{j}(-\boldsymbol{k}) M_{j, j} f_{j}(\boldsymbol{k})+\text { H.c. }+\cdots .
$$

Here $S_{j}$ denotes the strongly nested domain in the vicinity of $\boldsymbol{k}_{j}$ (see red regions in Fig. 1a). The ellipsis in equation (37) stands for the pairing terms in the remainder of the Brillouin zone. Using equation (10) to rewrite the $f$-operators in terms of the 
$\Psi$ fields, we find

$$
\Delta_{\alpha, \beta}^{\dagger}(\boldsymbol{k})=2 \Delta_{0} M_{j, j}\left(\phi_{j}^{*}(-\boldsymbol{k})\right)_{\alpha}\left(\phi_{j}^{*}(\boldsymbol{k})\right)_{\beta}, \quad \forall \boldsymbol{k} \in S_{j} .
$$

By construction, $H$ is time-reversal invariant, which means that

$$
\theta_{H} H(k) \theta_{H}^{-1}=H(-\boldsymbol{k}), \quad \theta_{H}=e^{i \varphi} i \sigma_{2} \mathcal{K}
$$

with $\mathcal{K}$ denoting complex conjugation and arbitrary $\varphi \in \mathbb{R}$. It is straightforward to show that time-reversal invariance of the pairing term is equivalent to

$$
e^{2 i \varphi} \sigma_{2} \Delta^{\dagger}(\boldsymbol{k}) \sigma_{2}=-\Delta(\boldsymbol{k})
$$

Using the phase conventions in equations (15) and (17) and writing $\Delta_{0}=\left|\Delta_{0}\right| \mathrm{e}^{i \rho}$, $\rho \in \mathbb{R}$, one finds that equation (40) is satisfied if

$$
\rho+\varphi= \pm \frac{\pi}{2}
$$

The matrix elements

$$
\delta_{j}:=\left\langle\phi_{j}\left(\boldsymbol{q}_{j}\right)\left|\theta_{H} \mathcal{K} \Delta^{\dagger}\left(\boldsymbol{q}_{j}\right)\right| \phi_{j}\left(\boldsymbol{q}_{j}\right)\right\rangle,
$$

where $\boldsymbol{q}_{j}$ is an arbitrary point on the jth Fermi surface, can now be readily evaluated. Choosing $\boldsymbol{q}_{j}=\boldsymbol{k}_{j}$, one finds

$$
\begin{aligned}
\delta_{j} & =2 i e^{i \varphi} \Delta_{0} M_{j, j}\left(\phi_{j}^{\dagger}\left(\boldsymbol{k}_{j}\right) \sigma_{2} \phi_{j}^{*}\left(-\boldsymbol{k}_{j}\right)\right)\left(\phi_{j}^{\dagger}\left(\boldsymbol{k}_{j}\right) \phi_{j}\left(\boldsymbol{k}_{j}\right)\right) \\
& =2 i e^{i \varphi} \Delta_{0} M_{j, j} \phi_{j}^{\dagger}\left(\boldsymbol{k}_{j}\right) \sigma_{2} \sigma_{1} \phi_{j}\left(-\boldsymbol{k}_{j}\right) \\
& =-2 i e^{i \varphi} \Delta_{0} M_{j, j} \phi_{j}^{\dagger}\left(\boldsymbol{k}_{j}\right) \phi_{j}\left(\boldsymbol{k}_{j}\right) \\
& = \pm 2\left|\Delta_{0}\right| M_{j, j},
\end{aligned}
$$

again exploiting equations (15) and (17) as well as, in the last line, equation (41). The invariant is thus given by

$$
N=\prod_{j} \operatorname{sign}\left(\delta_{j}\right)= \begin{cases}+1 \text { (trivial), } & \left|\gamma_{0}\right|>\left|\gamma_{3}\right| \\ -1 \text { (nontrivial }), & \left|\gamma_{0}\right|<\left|\gamma_{3}\right| .\end{cases}
$$

\section{References}

1. Ohtomo, A. \& Hwang, H. Y. A high-mobility electron gas at the $\mathrm{LaAlO}_{3} / \mathrm{SrTiO}_{3}$ heterointerface. Nature 427, 423-426 (2004).

2. Reyren, N. et al. Superconducting interfaces between insulating oxides. Science 317, 1196-1199 (2007).

3. Caviglia, A. D. et al. Electric field control of the $\mathrm{LaAlO}_{3} / \mathrm{SrTiO}_{3}$ interface ground state. Nature 456, 624-627 (2008).

4. Brinkman, A. et al. Magnetic effects at the interface between non-magnetic oxides. Nat. Mater. 6, 493-496 (2007).

5. Li, L., Richter, C., Mannhart, J. \& Ashoori, R. C. Coexistence of magnetic order and two-dimensional superconductivity at $\mathrm{LaAlO}_{3} / \mathrm{SrTiO}_{3}$ interfaces. Nat. Phys. 7, 762-766 (2011).

6. Bert, J. A. et al. Direct imaging of the coexistence of ferromagnetism and superconductivity at the $\mathrm{LaAlO}_{3} / \mathrm{SrTiO}_{3}$ interface. Nat. Phys. 7, 767-771 (2011).

7. Banerjee, S., Erten, O. \& Randeria, M. Ferromagnetic exchange, spin-orbit coupling and spiral magnetism at the $\mathrm{LaAlO}_{3} / \mathrm{SrTiO}_{3}$ interface. Nat. Phys. 9, 626-630 (2013).

8. Li, X., Liu, W. V. \& Balents, L. Spirals and skyrmions in two dimensional oxide heterostructures. Phys. Rev. Lett. 112, 067202 (2014).

9. Wollman, D. A., Van Harlingen, D. J., Lee, W. C., Ginsberg, D. M. \& Leggett, A. $J$. Experimental determination of the superconducting pairing state in YBCO from the phase coherence of YBCO-Pb dc SQUIDs. Phys. Rev. Lett. 71, 2134-2137 (1993).

10. Tsuei, C. C. et al. Pairing symmetry and flux quantization in a tricrystal superconducting ring of $\mathrm{YBa}_{2} \mathrm{Cu}_{3} \mathrm{O}_{7-\delta}$. Phys. Rev. Lett. 73, 593-596 (1994).

11. Hasan, M. Z. \& Kane, C. L. Colloquium: topological insulators. Rev. Mod. Phys. 82, 3045-3067 (2010).

12. Qi, X.-L. \& Zhang, S.-C. Topological insulators and superconductors. Rev. Mod. Phys. 83, 1057-1110 (2011).

13. Fu, L. \& Berg, E. Odd-parity topological superconductors: theory and application to $\mathrm{Cu}_{x} \mathrm{Bi}_{2} \mathrm{Se}_{3}$. Phys. Rev. Lett. 105, 097001 (2010).

14. Deng, S., Viola, L. \& Ortiz, G. Majorana modes in time-reversal invariant $s$-wave topological superconductors. Phys. Rev. Lett. 108, 036803 (2012).

15. Nakosai, S., Tanaka, Y. \& Nagaosa, N. Topological superconductivity in bilayer rashba system. Phys. Rev. Lett. 108, 147003 (2012).

16. Nakosai, S., Budich, J. C., Tanaka, Y., Trauzettel, B. \& Nagaosa, N. Majorana bound states and nonlocal spin correlations in a quantum wire on an unconventional superconductor. Phys. Rev. Lett. 110, 117002 (2013).

17. Zhang, F., Kane, C. L. \& Mele, E. J. Time-reversal-invariant topological superconductivity and Majorana Kramers pairs. Phys. Rev. Lett. 111, 056402 (2013).
18. Keselman, A., Fu, L., Stern, A. \& Berg, E. Inducing time-reversal-invariant topological superconductivity and fermion parity pumping in quantum wires. Phys. Rev. Lett. 111, 116402 (2013).

19. Santander-Syro, A. F. et al. Two-dimensional electron gas with universal subbands at the surface of $\mathrm{SrTiO}_{3}$. Nature 469, 189-193 (2011).

20. King, P. D. C. et al. Quasiparticle dynamics and spin-orbital texture of the $\mathrm{SrTiO}_{3}$ two-dimensional electron gas. Nat. Commun. 5, 3414 (2014).

21. Pentcheva, R. \& Pickett, W. E. Correlation-driven charge order at the interface between a mott and a band insulator. Phys. Rev. Lett. 99, 016802 (2007).

22. Pentcheva, R. \& Pickett, W. E. Ionic relaxation contribution to the electronic reconstruction at the $n$-type $\mathrm{LaAlO}_{3} / \mathrm{SrTiO}_{3}$ interface. Phys. Rev. B 78, 205106 (2008).

23. Michaeli, K., Potter, A. C. \& Lee, P. A. Superconducting and Ferromagnetic phases in $\mathrm{SrTiO}_{3} / \mathrm{LaAlO}_{3}$ oxide interface structures: possibility of finite momentum pairing. Phys. Rev. Lett. 108, 117003 (2012).

24. Joshua, A., Pecker, S., Ruhman, J., Altman, E. \& Ilani, S. A universal critical density underlying the physics of electrons at the $\mathrm{LaAlO}_{3} / \mathrm{SrTiO}_{3}$ interface. Nat. Commun. 3, 1129 (2012).

25. Fête, A., Gariglio, S., Caviglia, A. D., Triscone, J.-M. \& Gabay, M. Rashba induced magnetoconductance oscillations in the $\mathrm{LaAlO}_{3}-\mathrm{SrTiO}_{3}$ heterostructure. Phys. Rev. B 86, 201105(R) (2012).

26. Dresselhaus, G. Spin-Orbit Coupling Effects in Zinc Blende Structures. Phys. Rev 100, 580-586 (1955).

27. Rashba, E. I. Properties of semiconductors with an extremum loop. Cyclotron and combinational resonance in a magnetic field perpendicular to the plane of the loop. Sov. Phys. Solid State 2, 1109-1122 (1960).

28. Caviglia, A. D. et al. Tunable Rashba spin-orbit interaction at oxide interfaces. Phys. Rev. Lett. 104, 126803 (2010).

29. Shalom, M. B., Sachs, M., Rakhmilevitch, D., Palevski, A. \& Dagan, Y. Tuning spin-orbit coupling and superconductivity at the $\mathrm{SrTiO}_{3} / \mathrm{LaAlO}_{3}$ interface: a magnetotransport study. Phys. Rev. Lett. 104, 126802 (2010).

30. Bucheli, D., Grilli, M., Peronaci, F., Seibold, G. \& Caprara, S. Phase diagrams of voltage-gated oxide interfaces with strong Rashba coupling. Phys. Rev. B 89, 195448 (2014).

31. Zhong, Z., Toth, A. \& Held, K. Theory of spin-orbit coupling at $\mathrm{LaAlO}_{3} / \mathrm{SrTiO}_{3}$ interfaces and $\mathrm{SrTiO}_{3}$ surfaces. Phys. Rev. B 87, 161102(R) (2013).

32. Shankar, R. Renormalization-group approach to interacting fermions. Rev. Mod. Phys. 66, 129-192 (1994).

33. Chubukov, A. V., Efremov, D. V. \& Eremin, I. Magnetism, superconductivity, and pairing symmetry in iron-based superconductors. Phys. Rev. B 78, 134512 (2008).

34. Cvetkovic, V., Throckmorton, R. E. \& Vafek, O. Electronic multicriticality in bilayer graphene. Phys. Rev. B 86, 075467 (2012).

35. Richter, C. et al. Interface superconductor with gap behaviour like a hightemperature superconductor. Nature 502, 528-531 (2013).

36. Altland, A. \& Zirnbauer, M. R. Nonstandard symmetry classes in mesoscopic normal-superconducting hybrid structures. Phys. Rev. B 55, 1142-1161 (1997).

37. Ryu, S., Schnyder, A. P., Furusaki, A. \& Ludwig, A. W. W. Topological insulators and superconductors: tenfold way and dimensional hierarchy. New J. Phys. 12, 065010 (2010).

38. Bernevig, B. A. Topological Insulators and Topological Superconductors (Princeton University Press, Princeton, 2013).

39. Qi, X.-L., Hughes, T. L. \& Zhang, S.-C. Topological invariants for the Fermi surface of a time-reversal-invariant superconductor. Phys. Rev. B 81, 134508 (2010).

40. Yada, K., Onari, S., Tanaka, Y. \& Inoue, J. Electrically controlled superconducting states at the heterointerface $\mathrm{SrTiO}_{3} / \mathrm{LaAlO}_{3}$. Phys. Rev. B 80, 140509(R) (2009).

41. Xiang, Y.-Y., Wang, W.-S., Wang, Q.-H. \& Lee, D.-H. Topological superconducting phase in the vicinity of ferromagnetic phases. Phys. Rev. B 86, 024523 (2012).

42. Wang, L. \& Vafek, O. Unconventional superconductivity in a two-dimensional repulsive gas of fermions with spin-orbit coupling. Physica C 497, 6-18 (2014).

43. Mohanta, N. \& Taraphder, A. Possibility of Majorana bound states at LaAlO3/SrTiO3 interface. Preprint at http://arxiv.org/abs/1312.7779 (2013).

44. Fidkowski, L., Lutchyn, R. M., Nayak, C. \& Fisher, M. P. A. Majorana zero modes in one-dimensional quantum wires without long-ranged superconducting order. Phys. Rev. B 84, 195436 (2011).

45. Fidkowski, L., Jiang, H.-C., Lutchyn, R. M. \& Nayak, C. Magnetic and superconducting ordering in one-dimensional nanostructures at the $\mathrm{LaAlO}_{3} /$ $\mathrm{SrTiO}_{3}$ interface. Phys. Rev. B 87, 014436 (2013).

46. Kim, Y., Lutchyn, R. M. \& Nayak, C. Origin and transport signatures of spinorbit interactions in one- and two-dimensional $\mathrm{SrTiO}_{3}$-based heterostructures. Phys. Rev. B 87, 245121 (2013).

47. Milde, P. et al. Unwinding of a Skyrmion Lattice by Magnetic Monopoles. Science 340, 1076-1080 (2013).

48. Agterberg, D. F., Babaev, E. \& Garaud, J. Unconventional magnetic response of clean interface superconductors with strong Rashba spin-orbit coupling. Preprint at http://arxiv.org/abs/1403.6655 (2014) 
49. Ong, T. T. \& Coleman, P. ${ }^{3} \mathrm{He}-\mathrm{R}:$ A Topological $s \pm$ Superfluid with Triplet Pairing. Preprint at http://arxiv.org/abs/1402.7372 (2014).

50. Beenakker, C. W. J. Search for Majorana fermions in superconductors. Annu. Rev. Condens. Matter Phys. 4, 113-136 (2013)

51. Schneider, C. W., Thiel, S., Hammerl, G., Richter, C. \& Mannhart, J. Microlithography of electron gases formed at interfaces in oxide heterostructures. Appl. Phys. Lett. 89, 122101 (2006).

52. Cen, C. et al. Nanoscale control of an interfacial metal-insulator transition at room temperature. Nat. Mater. 7, 298-302 (2008).

53. Dahm, T., Manske, D., Fay, D. \& Tewordt, L. Isotope effect and resistivity in the Hubbard model for d-wave superconductivity. Phys. Rev. B 54, 12006-12009 (1996).

54. Nunner, T., Schmalian, J. \& Bennemann, K. H. Influence of electron-phonon interaction on spin-fluctuation-induced superconductivity. Phys. Rev. B 59, 8859-8868 (1999).

55. Honerkamp, C., Fu, H. \& Lee, D.-H. Phonons and $d$-wave pairing in the twodimensional Hubbard model. Phys. Rev. B 75, 014503 (2007).

56. Wan, X. \& Savrasov, S. Turning a band insulator into an exotic superconductor. Nat. Commun. 5, 4144 (2014).

57. Brydon, P. M. R., Hui, H.-Y. \& Sau, J. D. Odd-parity superconductivity from phonon-mediated pairing. Preprint at http://arxiv.org/abs/1402.7061.

\section{Acknowledgements}

We are grateful for discussions with S. Beyl, A.V. Chubukov, A.M. Finkel'stein, E.J. König, D. Mendler, A.D. Mirlin, and J.-M. Triscone. We acknowledge financial support by the Deutsche Forschungsgemeinschaft through grant SCHM 1031/4-1.

\section{Author contributions}

M.S. and J.S. contributed extensively to the calculations and wrote the paper.

\section{Additional information}

Supplementary Information accompanies this paper at http://www.nature.com/ naturecommunications

Competing financial interests: The authors declare no competing financial interests.

Reprints and permission information is available online at http://npg.nature.com/ reprintsandpermissions/

How to cite this article: Scheurer, M. S. et al. Topological superconductivity and unconventional pairing in oxide interfaces. Nat. Commun. 6:6005 doi: 10.1038/ ncomms7005 (2015). 